\title{
Estudio del metabolismo de los ácidos grasos a través de la investigación de las dietas cetogénicas. Aplicación de un ciclo de mejora en aula en la asignatura de Regulación del metabolismo
}

Study of metabolism of fatty acids through research on ketogenic diets. Application of classroom improvement cycle in the subject of metabolic regulation

MARÍA JOSÉ HUERTAS ROMERA

ORCID: https://orcid.org/0000-0002-6800-3270

Universidad de Sevilla. Departamento de

Bioquímica Vegetal y Biología Molecular

mihuertas@us.es

Fecha de recepción: 02/06/19

Fecha de aceptación: 15/07/19

DOI: http://dx.doi.org/10.12795/9788447221912.048

Pp.: 1109-1134 
Se presenta una experiencia de mejora en el aula en la asignatura de regulación del metabolismo en el Grado en Bioquímica. En este trabajo se propone el cambio en el modelo teórico habitual de clases magistrales a un modelo de investigación que implica el aprendizaje del metabolismo de los ácidos grasos y la regulación a través de la investigación sobre las dietas cetogénicas. Los alumnos han integrado conceptos que van desde la síntesis, degradación y movilización de ácidos grasos hasta el metabolismo de cuerpos cetónicos y colesterol. De esta manera se ha conseguido un modelo más participativo donde los estudiantes han elaborado e integrado los modelos de regulación que rigen este tipo de dietas. Los cuestionarios han sido una herramienta clave en este proceso ya que han permitido obtener una información importante de la evolución de los alumnos. Los resultados muestran una adquisición adecuada de los conceptos trabajados.

Palabras clave: Ácidos grasos, dietas cetogénicas, regulación del metabolismo, docencia universitaria, experimentación docente universitaria.

\section{Abstract}

A classroom improvement cycle in the subject of metabolic regulation in Biochemistry Degree are presented. We propose a change in theoretical model based in master classes to a research model that involves learning the metabolism of fatty acids and regulation through research on ketogenic diets. The students have integrated different concepts as the synthesis, degradation and mobilization of fatty acids and metabolism of ketone bodies and cholesterol. Thus, a more participatory model has been achieved where students have developed and integrated regulatory models that govern this type of diet. The questionnaires have been a key tool in this process since they have allowed obtaining important information about the evolution of the students. The results obtained show an adequate acquisition of the concepts that have been worked on.

Key words: Fatty acids, ketogenic diets, Metabolic regulation, Higher Education, learning in Higher education.

Jornadas de Formación e Innovación Docente del Profesorado | № 2 (2019) Esta obra se distribuye con la licencia Creative Commons Reconocimiento-NoComercial-SinObraDerivada Internacional (CC BY-NC-ND 4.0.) 


\section{Descripción del contexto}

En este trabajo se describe la propuesta y resultados de la aplicación de un ciclo de mejora en aula (CIMA) que se ha desarrollado en la asignatura denominada Regulación del metabolismo. Esta es una asignatura obligatoria de segundo del Grado de Bioquímica de la Facultad de Biología de la Universidad de Sevilla. El curso está compuesto por un grupo pequeño de alumnos (unos 60) que acceden al grado con una nota de corte elevada. En general son alumnos muy preparados y con buen nivel. La asignatura complementa una asignatura que cursan en el primer curso del grado denominada Fundamentos en Bioquímica. La asignatura de Regulación la impartimos entre dos profesores, una primera parte relacionada con el metabolismo del carbono y su regulación y una segunda parte que complementa a la primera y que añade metabolismo del nitrógeno e integración metabólica. Los alumnos en este curso tienen asignaturas muy complejas y durante el cuatrimestre donde se desarrolla mi asignatura tienen mucha carga académica. Esto condiciona que los alumnos no asistan a clase de manera continua debido a que mucha de la información se puede obtener en numerosos libros de texto (hay muchos manuales de metabolismo y regulación clásicos).

\section{Diseño previo del Ciclo de Mejora.}

La razón que originó la realización de un CIMA en esta asignatura estaba relacionada con fomentar la motivación de los alumnos hacia esta materia. En este sentido es importante, una vez tomada la decisión de llevar a cabo un cambio, meditar acerca de la metodología que nos permita cambiar de manera profunda la forma de impartir la docencia. El tema seleccionado para llevar a cabo el CIMA fue 
"Metabolismo de los ácidos grasos y su regulación". Este es un tema que está estructurado en dos bloques uno primero que abarca la síntesis y degradación de ácidos grasos y su regulación y otro bloque dedicado al metabolismo del colesterol. En el diseño previo se utilizó, como punto de comienzo, partir de una pregunta clave que generará curiosidad en los alumnos y cuya resolución nos permitiera ir avanzando en el proceso de aprendizaje (Bain, 2007). La pregunta inicial que se planteó fue: Las dietas con alto o bajo contenido en hidratos de carbono ¿son saludables?

\section{Mapa de contenidos}

Para responder a esta pregunta se elaboró un mapa de contenidos (Figura 1) en el que destacaron los contenidos conceptuales (azul), los procedimentales (rojo) y los actitudinales (verde). Interrelacionar los diferentes tipos de contenidos a través de un mapa de contenidos presenta una serie de ventajas: por un lado, permite una visión amplia de la asignatura y por el otro posibilita un mejor tratamiento de los problemas sociales además de que ayuda al desarrollo del espíritu crítico (García, Porlán y Navarro, 2017).

Jornadas de Formación e Innovación Docente del Profesorado | № 2 (2019) Esta obra se distribuye con la licencia Creative Commons 
Estudiodelmetabolismodelosácidosgrasosatravésdelainvestigacióndelasdietascetogénicas.AplicacióndeunciclodemejoraenaulaenlaasignaturadeRegulacióndelmetabolismo

\section{MARÍA JOSÉ HUERTAS ROMERA}

Las dietas con alto o bajo contenido en hidratos de carbono ¿Son saludables?

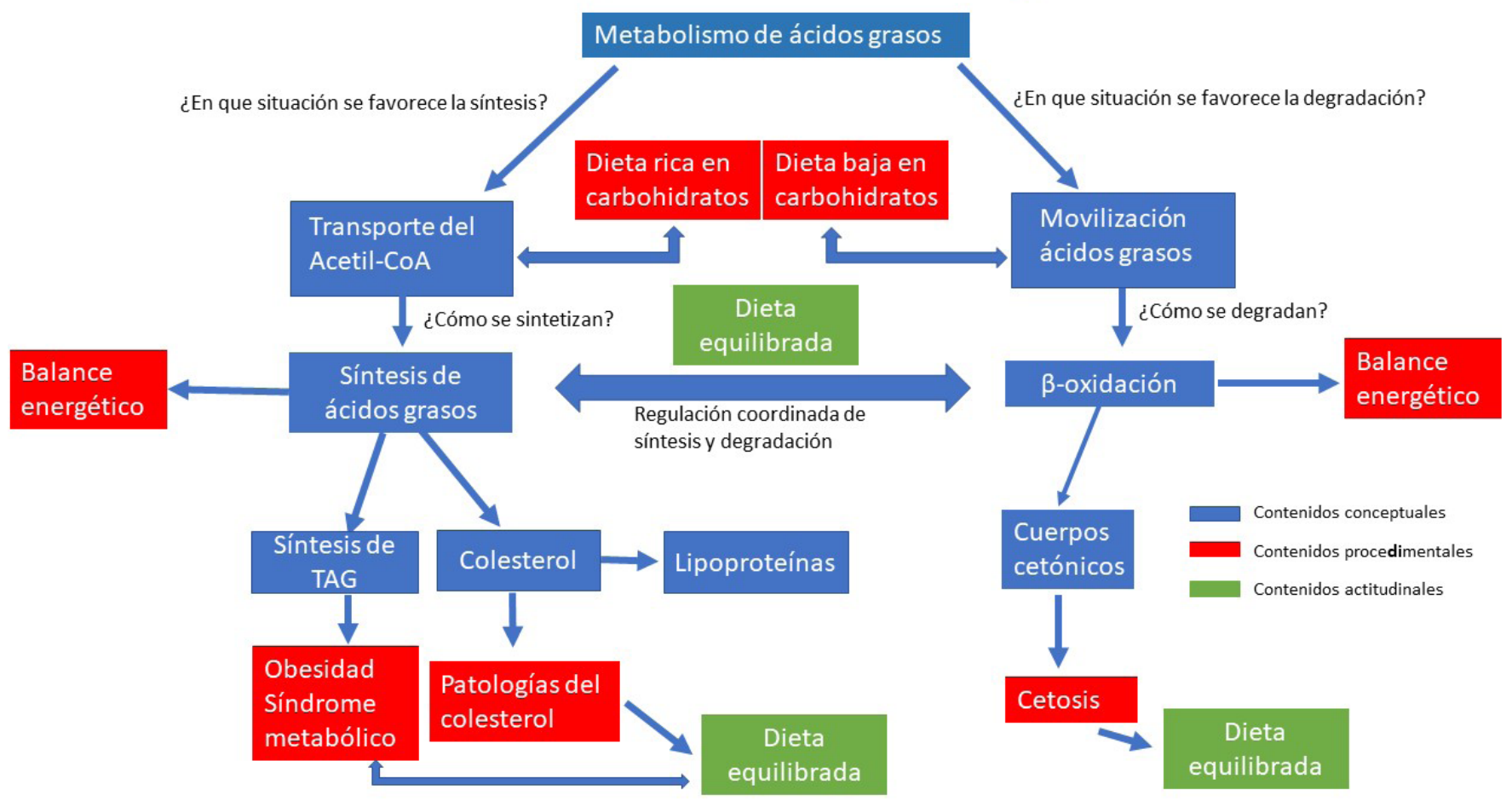

Figura 1. Mapa de contenidos del tema Metabolismo de ácidos grasos

Jornadas de Formación e Innovación Docente del Profesorado | № 2 (2019)

cc (i) E $\Theta$ Esta obra se distribuye con la licencia Creative Commons

In NC ND Rernacional (CC BY-NC-ND 4.0.) 
Como indique anteriormente. el mapa de contenidos se inició con una pregunta clave. A través del trabajo que implica generar respuestas a esta pregunta los estudiantes irán adquiriendo conceptos relacionados con la síntesis y degradación de ácidos grasos y su regulación. Además, aprenderán cómo este tipo de dietas conlleva un cambio metabólico en el que se favorece, a través de la regulación de enzimas clave, la síntesis de cuerpos cetónicos, la degradación del glucógeno y la degradación de proteínas. Asimismo, podrán analizar los cambios metabólicos qué ocurren si aumentamos la ingestión de hidratos de carbono. Los contenidos actitudinales están relacionados con la idea de la necesidad de llevar una dieta equilibrada que es importante para el buen funcionamiento del organismo. Los contenidos procedimentales permitirán estudiar aquellas situaciones patológicas derivadas de una mala alimentación. Como conclusión cabe destacar que la realización del mapa de contenidos permitirá reflexionar acerca de los contenidos que habitualmente se encuentran este tema de la asignatura, seleccionando aquellos que son conceptos claves.

\section{Secuencia de actividades}

Uno de los aspectos claves en la aplicación de un modelo metodológico implica el diseño de la secuencia de actividades de tal forma que permita detallar lo que va a ocurrir en el aula. La organización de una secuencia de actividades permite por un lado garantizar la coherencia entre el modelo posible y la intervención y por otra ser flexibles para adaptarse mejor a la evolución de los acontecimientos del aula (de Alba y Porlán, 2017). Para la aplicación del CIMA se diseñó la siguiente secuencia de actividades:

1. En la primera sesión se llevó a cabo la realización de un cuestionario inicial. Este estaba diseñado de tal forma que presentaba una serie 
de preguntas relacionadas con los contenidos conceptuales y algunos actitudinales del mapa de contenidos. El análisis de las respuestas de los cuestionarios permitió conocer del punto de partida de los alumnos. El cuestionario se realizó en clase a través de la plataforma socrative y fue anónimo.

2. El siguiente paso en la secuencia de actividades fue abordar el estudio de las dietas denominadas cetógenicas: que son dietas con muy poco aporte en hidratos de carbono y que durante mucho tiempo han estado de moda y otras dietas ricas en hidratos de carbono que es la que siguen muchas personas que hacen ejercicio fisico intenso o running. Se organizaron los alumnos en grupos de 3-4 estudiantes y se trabajó ahondando en las características de estas dietas desde el punto de vista, nutricional y metabólico.

3. En la plataforma se propuso una tarea para que la vayan realizando: toda la información que vamos obteniendo acerca de las dietas la pueden ir resumiendo en un documento y al final del tema elaborar un breve resumen contestando a la pregunta clave.

4. En las siguientes sesiones se llevó a cabo un estudio teórico, basándonos en el conocimiento previo de los estudiantes, de los procesos metabólicos que implican la movilización de los ácidos grasos, la oxidación de estos y la síntesis de cuerpos cetónicos. Nos centramos en la síntesis de cuerpos cetónicos. A través de los distintos datos obtenidos por los estudiantes se evaluó la eficacia de esta dieta basada en los conocimientos que hayan adquirido de metabolismo y regulación.

5. En la siguiente sesión, de la misma manera que en paso anterior, se abordó la investigación de una dieta rica en carbohidratos que utilizan los 
deportistas. Se estudiaron procesos de transporte del acetil CoA al citosol y la sintesis de ácidos grasos y los procesos que lo regulan. Posteriormente se abordó la síntesis de colesterol y se profundizará en los procesos patológicos asociados a un elevado depósito de ácidos grasos o colesterol.

6. Tras terminar las sesiones se hará un resumen de los diferentes tipos de dietas integrando los conceptos de metabolismo y regulación (finalización de la tarea propuesta en el punto 3) y se les pasará el cuestionario final para que vuelvan a rellenarlo con los conocimientos adquiridos.

En todo momento los conceptos actitudinales están dirigidos a definir de una manera critica una dieta saludable dependiendo del estilo de vía, patologías o características de los individuos y trabajando en todo momento los conceptos metabólicos. Los contenidos procedimentales están relacionados con las patologías que se derivan de la aplicación de una u otra dieta.

\section{Cuestionario inicial y final}

Una de las tareas más complejas es conocer que saben y que aprenden los estudiantes realmente. Uno de los instrumentos que se utilizan para obtener esta información son los cuestionarios (Rivero y Porlán, 2017). El cuestionario está basado en el mapa de contenidos ya que de esta forma tenemos seleccionados los contenidos o aspectos más relevantes. El cuestionario que diseñamos se desarrolló en la plataforma socrative para facilitar el acceso por parte d ellos estudiante y el análisis posterior.

1. ¿La Movilización de las grasas es un proceso regulado? Explica brevemente tu respuesta

2. ¿En qué orgánulo se lleva a cabo la degradación de ácidos grasos? ¿Qué proceso implica la activación de los mismos? 
3. ¿Existe alguna diferencia entre la degradación de ácidos grasos de cadena par e impar?

4. Razona esta afirmación: Los cuerpos cetónicos se producen sólo en determinadas situaciones metabólicas

5. ¿Crees que los cuerpos cetónicos pueden ser un combustible? ¿Cómo?

6. La síntesis de ácidos grasos implica un conjunto de reacciones inversas a la degradación ¿Es correcto? Razona brevemente tu respuesta.

7. En la regulación de la sintesis y degradación de ácidos grasos

a) La acetil CoA carboxilasa juega un papel importante en la regulación

b) La sintesis está regulada pero no la degradación

c) La acetil CoA carboxilasa está regulada a nivel hormonal y por modificación covalente

d) Las respuestas $A$ y $C$ son correctas

8. Explica brevemente que ocurriría si un individuo tuviese una dieta baja en grasas durante tiempo prolongado

9. ¿Qué es el HDL o colesterol bueno?

10. ¿Conoces si la degradación de colesterol está regulada?

\section{Aplicación del Ciclo mejora}

\section{Relato resumido de las sesiones}

En la primera sesión en la clase se describió cómo se iba a impartir el tema y que iba a ser muy diferente a como se había trabajado anteriormente. El estudio de los diferentes contenidos lo íbamos a llevar a cabo a través del análisis de diferentes tipos de dietas. Lo primero fue evaluar de donde partimos para ver los conocimientos 
que tienen acerca de conceptos clave del metabolismo de ácidos grasos. Para ello pasé el cuestionario inicial que realizaron en 15 minutos. Noté que algunos alumnos presentaban una actitud negativa, ya que pensaban que están perdiendo el tiempo. Esa sensación la experimenté a lo largo de todo el ciclo de mejora ya que estos alumnos tienen muchas tareas y están muy agobiados. La cuestión o preocupación fundamental que tenían los estudiantes inicialmente es si los cuestionarios iban a tener un peso en la evaluación o no. Para evitar esta preocupación les comuniqué que los cuestionarios podían ir con seudónimo y que no iba a evaluar la actividad. En la primera sesión hice grupos de tres personas para que trabajaran juntos y buscaran información general de dietas tanto cetogénicas, con baja cantidad de hidratos de carbono, como dietas ricas en hidratos de carbono. Los dejé trabajar durante un tiempo y pasado ese tiempo resumimos en la pizarra las características de las diferentes dietas. Ese fue el punto de partida. La secuencia de actividades resumida, derivada de la descrita anteriormente, fue: i) Presentación de conceptos y pregunta clave, ii) Actividad practica de búsqueda de información en grupo y iii) Puesta en común y debate. El problema fundamental en la primera sesión la tuve con el tiempo que destiné a la actividad de investigación y la organización de la información.

El análisis inicial del cuestionario nos permitió situar a los estudiantes en el punto de partida y además llevar a cabo cambios o ajustes en el mapa de contenidos (Rivero y Porlán, 2017). Cabe destacar que dominan muchos conceptos de manera general acerca del metabolismo, otros conceptos no los saben o tienen ideas erróneas. El cuestionario también nos permitió detectar determinados obstáculos en el aprendizaje que deben de tenerse en cuenta en la aplicación del ciclo. Como ejemplo, el hecho de que no recuerdan los procesos de sintesis y degradación de ácidos grasos o no conocen la regulación de estos procesos. En las siguientes clases se trabajó siguiendo la misma 
secuencia de actividades descrita anteriormente y transcurrieron con normalidad. En cada clase íbamos trabajando cada una de las cuestiones del cuestionario ahondando en un nivel mayor de complejidad para obtener un conocimiento claro de estos conceptos. A su vez íbamos trabajando con la idea de la integración metabólica a través del estudio de las dietas. Aprovechando la parada de semana santa les plantee un trabajo voluntario en el que hicieran un resumen de todos los conceptos trabajados en clase, investigación e integración acerca de las dietas.

En la primera sesión, después de vacaciones, encontré mucha dificultad para que los estudiantes volvieran al ritmo de trabajo en grupo por lo que reanudamos las actividades haciendo un repaso de la regulación de la degradación y cuerpos cetónicos, conceptos claves para la evaluación de las dietas. Repasamos también la síntesis de ácidos grasos que no recordaban nada y los llevo a investigar este proceso en relación con nuestra actividad de las dietas. Con respecto a la actividad voluntaria planteada únicamente algunos alumnos me enviaron la tarea propuesta y al conocerlo el resto la pregunta fundamental es si va a ser o no evaluado. En la siguiente sesión como hemos repasado muchos conceptos clave y hemos ido aumentando la complejidad del conocimiento les propongo hacer un repaso de las rutas glucolíticas que habían visto anteriormente para integrar el metabolismo de las grasas. Ya disponen de información y datos importantes para que vayamos elaborando un diagrama crítico. En esta ocasión han participado bastante y tienen mucho interés en integrar el metabolismo con la dieta. Las siguientes sesiones hablamos del metabolismo del colesterol y de la regulación y como parte final les pase de nuevo los cuestionarios iniciales. Solo 13 alumnos realizaron los cuestionarios iniciales y finales, el resto que son 18 no asistieron a clase por un parcial que estaban preparando y solo un par de ellos no habían realizado el cuestionario inicial. El último día de la sesión analice con ellos el cuestionario 
y llegamos a algunas conclusiones de algunos conceptos que debían de afianzarse (nuevos obstáculos no resueltos). En concreto:

1. Importante trabajar el concepto de movilización de ácidos grasos y distinguirlo de oxidación o sintesis.

2. La idea de la sintesis de cuerpos cetónicos solo en situación de ayuno prolongado. Es una idea incorrecta y la trabajamos de nuevo.

3. La última pregunta del cuestionario es una pregunta trampa ya que no hay degradación de colesterol...reflexionamos acerca de cómo tenemos que leer las preguntas y analizar muy bien que se pregunta. Hace falta tener muy claro el concepto de síntesis de colesterol y su regulación.

4. Repasamos de nuevo como se transportan los lípidos a través del plasma en lipoproteínas LDL y HDL. Afianzamos las diferencias entre estas dos lipoproteínas.

5. La pregunta acerca de la sintesis y degradación de ácidos grasos (cuestión número 6) es otra que puede llevar a confusión, así que la analizamos de nuevo.

De manera general la evaluación del cuestionario al final del ciclo fue muy positiva ya que habían adquirido muchos conceptos de manera clara y precisa y a un buen nivel de complejidad.

Además, comentamos la actividad voluntaria de las dietas que habían trabajado unos cuantos alumnos y resulto una actividad complementaria muy interesante. 


\section{Evaluación del aprendizaje de los estudiantes}

\section{Escaleras de aprendizaje}

Una vez aplicado el cuestionario inicial y final es preciso clasificar y analizar las respuestas de manera sistemática de tal forma que nos permita conocer el grado de evolución de la clase (Rivero y Porlán, 2017). Para ello se compararon los resultados de los dos cuestionarios que contenían las preguntas que se habían trabajado durante el curso y se plasmaron en las escaleras de aprendizaje. Como se ha indicado anteriormente el cuestionario inicial nos mostró que muchos alumnos tienen algunos conocimientos previos sobre el tema y que son capaces de establecer un razonamiento científico importante. Son alumnos de segundo que han cursado una asignatura bioquímica y tienen conceptos de metabolismo claros. En este ciclo de mejora se han analizado una muestra de 13 cuestionarios individuales de los 31 que se recogieron.

Las respuestas las subdividi en tres grandes grupos.

1. Respuesta correcta acercándose bastante a lo que espero.

2. Respuesta correcta pero el alumno no tiene claro la respuesta completa o divaga en algunas partes de esta.

3. Respuesta incorrecta.

Se muestran las escaleras de aprendizaje correspondientes a algunas de las cuestiones más representativas en azul se indica el resultado de los cuestionarios iniciales y en rojo los finales:

Jornadas de Formación e Innovación Docente del Profesorado | № 2 (2019) Esta obra se distribuye con la licencia Creative Commons Reconocimiento-NoComercial-SinObraDerivada 
4. Razona esta afirmación: Los cuerpos cetónicos se producen sólo en determinadas situaciones metabólicas

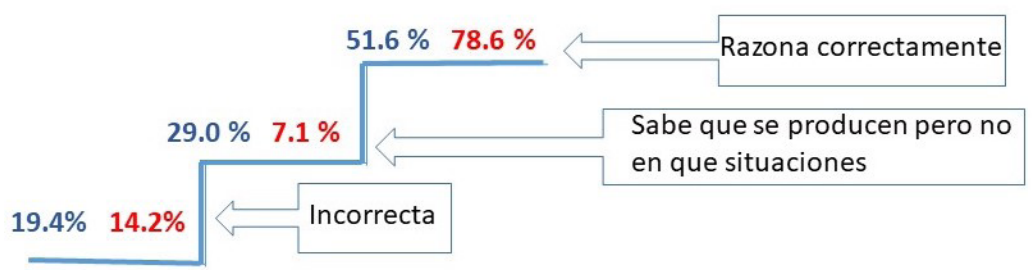

Figura 2. Escalera de aprendizaje de la pregunta 4

En la Figura 2 se muestra el resultado de la escalera de aprendizaje para la pregunta 4. La mayor parte de los estudiantes partían de la idea de que esta afirmación es correcta pero no sabían bien razonarla (29\%), un 52\% conocía la respuesta correcta y solo un $20 \%$ no sabía nada. En la prueba final la mayor parte de los alumnos que no saben o en que situaciones se producen terminan teniendo claro que el proceso tiene lugar en condiciones de ayuno y ayuno prolongado, así como algunos adquieran la idea de que también se lleva a cabo el proceso en condiciones energéticamente favorables (78,6\%).

5. ¿Crees que los cuerpos cetónicos pueden ser un combustible? ¿Cómo?

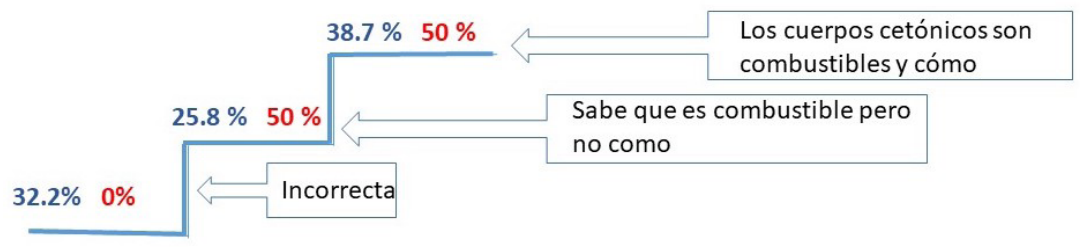

Figura 3. Escalera de aprendizaje de la pregunta 5

En la figura 3 se muestra la escalera de aprendizaje de la pregunta 5. Al inicio muchos de los estudiantes no conocen que los cuerpos cetónicos pueden ser un combustible, si no que lo relacionan con un producto tóxico derivado de una situación de ayuno prolongado o diabetes 
incontrolada (32,2\%), algunos saben que pueden usarlos el cerebro $(25,8 \%)$ y otro porcentaje de la clase saben la respuesta correcta. Tras el ciclo a la mitad de los alumnos les queda claro que son combustibles lo que queda por determinar es como se lleva a cabo la utilización, la mitad no lo recordaba (50\%) y si la otra mitad (50\%).

6. La síntesis de ácidos grasos implica un conjunto de reacciones inversas a la degradación ¿Es correcto? Razona brevemente tu respuesta.

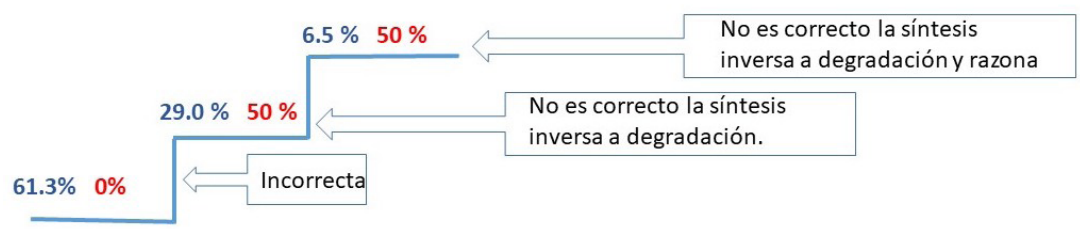

Figura 3. Escalera de aprendizaje de la pregunta 6

En la Figura 3 se muestra la escalera de aprendizaje de la pregunta 6 . En esta cuestión la mayoría opina que la frase es correcta (61\%). Esta idea es incorrecta ya que son procesos totalmente diferentes, aunque se puede pensar inicialmente que son procesos inversos. Al final del ciclo a la mitad de los estudiantes les queda claro que no son procesos inversos (50\%) pero no razonan porque y otra mitad (50\%) son capaces de razonar acerca de las diferencias en el proceso.

La evolución en general en todas las preguntas ha sido positiva. En la tabla 1 se muestran los resultados analizando si los alumnos no han avanzado nada (no han subido ningún escalón), han avanzado un escalón de conocimiento (suben un escalón) o ya estaban en un nivel de conocimiento superior.

Jornadas de Formación e Innovación Docente del Profesorado I № 2 (2019) Esta obra se distribuye con la licencia Creative Commons Reconocimiento-NoComercial-SinObraDerivada 
Tabla 1. Porcentaje de alumnos que pasó a un nivel superior al de partida en la escalera de conocimiento tras el cuestionario final. (* dos alumnos descendieron un nivel)

\begin{tabular}{|l|l|l|l|l|l|l|l|l|l|l|}
\hline Preguntas & 1 & 2 & 3 & 4 & 5 & 6 & $7^{*}$ & 8 & 9 & 10 \\
\hline $\begin{array}{l}\text { No sube de } \\
\text { escalón \% }\end{array}$ & 21,4 & 28,6 & 21,4 & 14,3 & 7,2 & 42,9 & 14,3 & 28,6 & 7,2 & 14,3 \\
\hline $\begin{array}{l}\text { Subió un } \\
\text { escalón \% }\end{array}$ & 64,3 & 50 & 64,3 & 71,4 & 78,6 & 50 & 0 & 42,9 & 71,4 & 85,7 \\
\hline $\begin{array}{l}\text { Ya estaba en } \\
\text { escalón } \\
\text { superior \% }\end{array}$ & 14,3 & 21,4 & 14,3 & 14,3 & 14,3 & 7,2 & 78,6 & 28,6 & 21,4 & 0 \\
\hline
\end{tabular}

Si analizamos esta tabla destacamos que en todas las cuestiones la mayoría de los alumnos subió un escalón con respecto al conocimiento inicial. La observación de que hay un porcentaje de alumnos que no suben de escalón nos permite detectar las limitaciones del método y que es lo que hay que trabajar más a fondo en los temas siguientes. Destaco el hecho que un porcentaje pequeño ya estaba en ese escalón superior, dándonos idea de que estos estudiantes parten de conceptos fijados en otras asignaturas y que permite establecer un buen nivel para un aprovechamiento mucho más a fondo de la asignatura.

\section{Evaluación del Ciclo de Mejora}

El modelo metodológico desarrollado durante este ciclo de mejora ha sido fruto de la reflexión a partir de mi participación en el Curso General de Docencia Universitaria. El ciclo de mejora en general ha resultado muy satisfactorio, la idea de reconducir el metabolismo de los ácidos grasos a través del estudio de las dietas es muy interesante ya que permite ir generando conocimientos metabólicos y de regulación a través del análisis de estas dietas.

Como punto de partida de un programa de mejora las dificultades encontradas han sido las derivadas del cambio de modelo de docencia. Los cambios en los modelos 
docentes son procesos complejos que implican el ajuste del modelo metodológico dependiendo del grupo de alumnos y de su implicación en este proceso. La participación del alumnado es otro punto que necesita una mejora ya que es necesario el compromiso de los estudiantes en su propio aprendizaje (Bain, 2007) debido fundamentalmente a que están habituados a una enseñanza unidireccional de clases magistrales y genera desconcierto el cambio de modelo. La mayor dificultad ha sido la poca implicación de los estudiantes para los trabajos en grupo. Esta dificultad se puede solucionar teniendo en cuenta tanto la participación, como la realización de actividades o tareas relacionadas en la evaluación de la asignatura.

Una de las herramientas que más información ha aportado ha sido el uso de los cuestionarios, de manera inicial ha permitido detectar los conceptos que conocen los estudiantes y el nivel de complejidad y así ajustar el mapa de contenidos y la secuencia de actividades necesarias para alcanzar los objetivos o competencias que necesito trabajar. El análisis del cuestionario final ha permitido evaluar lo que han aprendido e incluso las partes que quizás no se han trabajado con intensidad o yo no se han desarrollado adecuadamente. Los alumnos han reforzado muy positivamente el trabajo de analizar los cuestionarios al principio y al final porque muchos de los conceptos claves han quedado muy claros. Además, la integración metabólica a través del estudio de las dietas ha generado un gran interés y una aplicación práctica de procesos que tienen una gran base teórica.

\section{Cuestiones que mantener y cambios a introducir}

La realización del curso General de Docencia Universitaria me ha planteado el cambio en la forma de dirigir mis clases. Respecto a la asignatura completa de Regulación de Metabolismo me planteo mantener una serie de 
cuestiones: el comienzo de cada tema con una cuestión que suponga un desafio es clave. Este tipo de cuestiones permiten despertar el interés del alumno y mantener ese interés a lo largo del tema. Además, fomenta la participación del alumnado en el proceso docente. Existen muchas herramientas que pueden dirigir el proceso de aprendizaje alrededor de la pregunta clave, algunas de ellas las he utilizado y las seguiré utilizando como videos, recortes de periódico con noticias actuales o artículos en revistas de prestigio, etc. El uso de los cuestionarios como herramienta es otra cuestión que voy a mantener en los temas, para ello y para no hacer muchos voy a hacer bloques que me permita agrupar conceptos.

Con respecto a los cambios a introducir están relacionados fundamentalmente con la mejora de algunos procesos que me permitirán llevar las clases más ordenadas. Trabajar en los mapas de contenidos es uno de los aspectos más importantes, en este sentido la selección de los contenidos a impartir es el proceso más complejo ya que hay que seleccionar los contenidos claves de otros y por lo tanto jerarquizar. Otro cambio importante es, una vez establecido el mapa de contenidos, trabajar en una secuencia de actividades que permita guiar el proceso de aprendizaje. Es importante que en cada momento esté muy bien establecido como se va a ir desarrollando cada sesión, aunque también es importante que de vez en cuando sucedan cosas inesperadas. Una buena secuencia de actividades, pienso que, permitirá esta flexibilidad. Por último, uno de los cambios importantes debe venir de la necesidad de evaluar el proceso de aprendizaje en cada uno de los pasos de la secuencia de actividades (calificar el trabajo en grupo, la exposición, discusión) es importante para el proceso de aprendizaje y para la motivación de los alumnos. 


\section{Principios didácticos que guiaran la docencia en el futuro}

Basándome en la lectura del capítulo ¿Cómo dirigen la clase? (Bain 2007) y el capítulo dedicado a la metodología de la enseñanza (De alba y Porlán, 2017) pretendo aplicar un modelo metodológico basado en el principio de investigación guiado por una cuestión desencadenante, enunciado de hipótesis, contraste y establecimiento de conclusiones. Para ello los principios didácticos serian:

- En cada bloque temático seleccionar tareas que despierten la curiosidad. Para ello se iniciará con una cuestión o formulación de un problema. La idea de dirigir la clase a través de una pregunta clave causa un cambio importante en el desarrollo de la docencia. Los alumnos se sienten más implicados y la clase es mucho más participativa.

- Relacionado con la idea de comenzar conociendo donde están los estudiantes el diseño de un cuestionario inicial es clave. Se pretende al inicio de cada tema global comenzar con un cuestionario de conocimientos donde se evaluará el punto de partida de los estudiantes. El mismo cuestionario al final permitirá evaluar cómo ha ido el proceso de aprendizaje.

- El diseño de un mapa de contenidos y una secuencia de actividades es también fundamental para guiar de manera ordenada el proceso de aprendizaje. Partiendo de la cuestión desencadenante y teniendo clara los contenidos que queremos enseñar, la secuencia nos permitirá guiar a los estudiantes por el proceso. En esta secuencia de actividades se plantearán actividades de trabajo autónomo y en grupo. Esto llevará a generar un entorno idóneo para el aprendizaje critico natural (Bain, 2007). Además, implica el compromiso del estudiante hacia la asignatura y el proceso de aprendizaje. En este sentido es importante permitir a los alumnos que dispongan de tiempo suficiente para el desarrollo de un razonamiento crítico. 
- Establecer sistemas de evaluación diversos (informes de trabajo, carpetas de aprendizaje, observación.) que permitan calificar las diferentes actividades en un proceso continuo. 


\section{Bibliografía}

Bain, K. (2007). Lo que hacen los mejores profesores universitarios. Valencia: Servicio de Publicaciones Universidad de Valencia.

Finkel, D (2008). Dar clases con la boca cerrada. Valencia: Servicio de Publicaciones Universidad de Valencia.

García, E., Porlán, R y Navarro (2017). Los fines y los contenidos. En Porlán, R. (Coord). Enseñanza Universitaria. Cómo mejorarla. pp. 55-72. Madrid: Ediciones Morata.

De Alba, N. Porlán, R (2017). La metodología de enseñanza. En Porlán, R. (Coord). Enseñanza Universitaria. Cómo mejorarla. pp. 37-53. Madrid: Ediciones Morata.

Rivero, A. y Porlan, R (2017). La evaluación en la enseñanza universitaria. En Porlán, R. (Coord). Enseñanza Universitaria. Cómo mejorarla. pp. 73-91. Madrid: Ediciones Morata. 


\section{2}

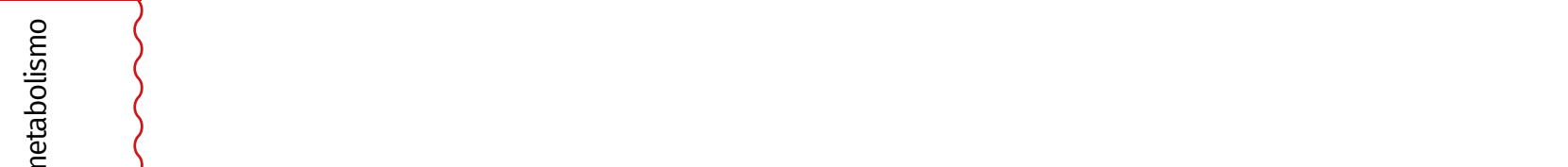


